\title{
Effects of histone deacetylase inhibitors on ATP-binding cassette transporters in lung cancer A549 and colorectal cancer HCT116 cells
}

\author{
HAO WANG ${ }^{1}$, CHUN-HUA CHI $^{2}$, YING ZHANG ${ }^{2}$, BIN SHI $^{3}$, RU JIA ${ }^{4}$ and BEN-JUN WANG ${ }^{2}$ \\ ${ }^{1}$ Department of Clinical Laboratory, The First Affiliated Hospital of University of Science and Technology of China, Hefei, \\ Anhui 230001; ${ }^{2}$ Department of Anorectal Surgery, Affiliated Hospital of Shandong University of Traditional Chinese \\ Medicine, Jinan, Shandong 250014; ${ }^{3}$ Anorectal Department of Traditional Chinese Medicine, The First Affiliated \\ Hospital of University of Science and Technology of China; ${ }^{4}$ Department of Anorectal Surgery, The Second \\ Affiliated Hospital of Anhui University of Chinese Medicine, Hefei, Anhui 230001, P.R. China
}

Received April 25, 2018; Accepted March 21, 2019

DOI: $10.3892 / 01.2019 .10319$

\begin{abstract}
Histone deacetylase (HDAC) inhibitors and DNA alkylators are effective components used in combination chemotherapy. In the present study, the effects of HDAC inhibitors on the expression of ATP-binding cassette (ABC) transporters were investigated. It was observed that HDAC inhibitors induced the expression of multidrug-resistant $\mathrm{ABC}$ transporters differently in lung cancer A549 cells than in colorectal cancer HCT116 cells. In these two cell lines, the HDAC inhibitors suberoylanilide hydroxamic acid (SAHA) and trichostatin A (TSA) significantly increased ABCB1 expression at the mRNA and protein levels, whereas they had no evident effect on ABCG2 protein expression. SAHA and TSA decreased ABCG2 mRNA expression in A549 cells and had no evident effect on ABCG2 mRNA expression in HCT116 cells. Notably, SAHA and TSA increased the mRNA expression levels of ABCC5, ABCC6, ABCC10, ABCC11 and $\mathrm{ABCC} 12$, as well as the protein expression levels of $\mathrm{ABCC} 2$, $\mathrm{ABCC} 10$ and $\mathrm{ABCC} 12$. By contrast, these inhibitors decreased the mRNA expression levels of $\mathrm{ABCC} 1, \mathrm{ABCC} 2, \mathrm{ABCC} 3$ and $\mathrm{ABCC} 4$, as well as the expression of $\mathrm{ABCC} 1$ and $\mathrm{ABCC} 3$ proteins. Furthermore, SAHA and TSA were found to downregulate HDAC3 and HDAC4, but not HDAC1 and HDAC2. Taken together, the results suggested that HDAC inhibitors work synergistically with DNA alkylators, in part, due to the inhibitory effect of these inhibitors on ABCC1 expression, which translocates these alkylators from inside to outside of
\end{abstract}

Correspondence to: Dr Ben-Jun Wang, Department of Anorectal Surgery, Affiliated Hospital of Shandong University of Traditional Chinese Medicine, 42 Wenhua West Road, Jinan, Shandong 250014, P.R. China

E-mail:wbj6688@163.com

Key words: histone deacetylase inhibitors, ATP-binding cassette transporter, ABCB1, ABCCs, ABCG2, multidrug resistance cancer cells. These results further suggested the possibility of antagonism when HDAC inhibitors are combined with anthracyclines and other ABCB1 drug ligands in chemotherapy.

\section{Introduction}

Multidrug resistance (MDR) occurs when cancer cells are resistant to a number of functionally and structurally different chemotherapeutic agents, and represents a major obstacle for successful treatment of cancer $(1,2)$. Efflux of anticancer drugs by ATP-binding cassette (ABC) transporters serves a crucial role in the development of the MDR phenotype (3-5). ABC transporters associated with chemoresistance include MDR-1, MDR-associated proteins and breast cancer resistance protein, which are coded for by $\mathrm{ABCB} 1, \mathrm{ABCC}$ and $\mathrm{ABCG} 2$, respectively (3). ABCB1 is the most well-known $\mathrm{ABC}$ transporter, and is able to extrude natural toxins, anticancer drugs and drug metabolites across the plasma membrane to confer an MDR phenotype in cancer cells (6-8). Similarly, ABCC members have been reported to confer chemoresistance in cancer cells by translocating a variety of structurally diverse glutathione conjugates or therapeutic drugs $(4,9)$. For instance, ABCC1, $\mathrm{ABCC} 2$ and $\mathrm{ABCC} 3$ cause resistance to hydrophobic anions, including several natural compounds, whereas ABCC4, ABCC5 and ABCC11 efflux cyclic nucleotides $(10,11)$. ABCG2, another MDR-associated protein, is a ubiquitous $\mathrm{ABC}$ transporter with an important role in the distribution, absorption and elimination of its substrate $(12,13)$. Collectively, these ABC transporters confer the MDR phenotype to cancer cells by reducing the intracellular concentrations of anticancer drugs to a nontoxic level. Furthermore, these ABC transporters are highly expressed in a number of human tumors and are often associated with poor prognosis $(14,15)$.

Histone deacetylase (HDAC) inhibitors have emerged as a novel class of anticancer agents due to their significant anticancer activities, including angiogenesis inhibition, and the promotion of cell cycle arrest, differentiation and apoptosis $(16,17)$. HDAC inhibitors may serve as potent anticancer drugs due to their broad antitumor activity and low toxicity in 
normal cells (18). Several HDAC inhibitors, including trichostatin A (TSA), suberoylanilide hydroxamic acid (SAHA; also known as vorinostat) and sodium butyrate, have exhibited potent anticancer activities in various cancer cells (19). SAHA has been approved by the US Food and Drug Administration as a treatment for cutaneous T-cell lymphoma (20). Furthermore, HDAC inhibitors have been demonstrated to work synergistically with a variety of antitumor agents, including gemcitabine, doxorubicin, etoposide, paclitaxel and cisplatin (21).

Our previous study and other previous research have demonstrated that treatment of cancer cells with HDAC inhibitors increases the expression of $\mathrm{ABCB} 1$, which results in the development of an MDR phenotype $(22,23)$. However, the effects of HDAC inhibitors on other MDR-associated $\mathrm{ABC}$ transporters have not previously been reported. Thus, the aim of the present study was to investigate the effects of two HDAC inhibitors, namely SAHA and TSA, on the expression levels of ABCB1, ABCCs and ABCG2 in lung cancer A549 and colorectal cancer HCT116 cells. The results indicated that HDAC inhibitors are able to induce differential expression of these $\mathrm{ABC}$ transporters. The present study suggests that more attention should be paid to drug combinations with HDAC inhibitors, as $\mathrm{ABC}$ transporters have different substrates. DNA alkylators are substrates of $\mathrm{ABCC} 1$. A decrease in $\mathrm{ABCC} 1$ protein level may contribute to the synergism of HDAC inhibitors and DNA alkylating agents. Conversely, HDAC inhibitors may antagonize the efficacy of anticancer drugs that are substrates of ABCB1 (14). These differential effects of HDAC inhibitors on the expression levels of drug transporters support the necessity for caution in combining these drugs with other chemotherapeutic agents.

\section{Materials and methods}

Chemicals and reagents. Oxaliplatin, 5-fluorouracil, SAHA and TSA were purchased from Sigma-Aldrich (Merck KGaA, Darmstadt, Germany). Primary antibodies against the following were purchased: ABCB1 (cat. no. 13978), purchased from Cell Signaling Technology, Inc. (MA, Danvers, USA); $\alpha$-tubulin (cat. no. sc-134237), obtained from Santa Cruz Biotechnology, Inc. (Dallas, TX, USA); ABCC2 (cat. no. 24893-1-AP), ABCC5 (cat. no. 19503-1-AP) and ABCC6 (cat. no. 27848-1-AP) obtained from ProteinTech Group, Inc. (Chicago, IL, USA); ABCC1 (cat. no. BS7474) and ABCG2 (cat. no. BS3482) from Bioworld Technology Co., Ltd. (Nanjing, China); ABCC3 (cat. no. ab3375), ABCC4 (cat. no. ab77184), ABCC10 (cat. no. ab91451), ABCC11 (cat. no. ab98979) and ABCC12 (cat. no. ab91453) from Abcam (Cambridge, MA, USA); HDAC1 (cat. no. ET1605-35), HDAC2 (cat. no. ET1607-78), HDAC3 (cat. no. ET1610-5) and HDAC4 (cat. no. ET1612-51), purchased from HuaAn Biotechnology Co., Ltd. (Hangzhou, China). Horseradish peroxidase (HRP)-conjugated secondary antibody (cat. nos. SH001X, SH002X and SH003X) were also purchased from DingGuo Biotechnology Co.,Ltd. (Guangzhou, China). The PrimeScript ${ }^{\circledR}$ RT reagent and SYBR ${ }^{\circledR}$ Premix Ex $\mathrm{Taq}^{\mathrm{TM}}$ kits were purchased from Takara Bio, Inc. (Otsu, Japan).

Cell culture. A549 and HCT116 cells were obtained from the Type Culture Collection of the Chinese Academy of Sciences (Shanghai, China). HCT116 cells were maintained in Dulbecco's modified Eagle's medium/F12 culture medium (Gibco; Thermo Fisher Scientific, Inc., Waltham, MA, USA) supplemented with $10 \%$ fetal bovine serum (FBS). A549 cells were cultured in RPMI-1640 culture medium (Gibco; Thermo Fisher Scientific, Inc.) supplemented with $10 \%$ FBS. The two cell lines were cultured in an incubator at $37^{\circ} \mathrm{C}$ with an atmosphere of $5 \% \mathrm{CO}_{2}$.

Reverse transcription-quantitative polymerase chain reaction $(R T-q P C R)$ detection. A549 and HCT116 cells were treated with SAHA $(0.5 \mu \mathrm{M})$ or TSA $(100 \mathrm{nM})$ at $37^{\circ} \mathrm{C}$ for $24 \mathrm{~h}$. Treatment with DMSO (equal volume added) was used as the control. Total mRNA was extracted from A549 and HCT116 cells using TRIzol reagent. RNA sample concentration was measured using a UV spectrophotometer, and optical density (OD)260/OD280 was limited to 1.8-2.0. A total of 500 ng RNA was used for cDNA synthesis with the PrimeScript ${ }^{\circledR}$ RT reagent kit. Subsequently, qPCR was performed on an ABI 7500 Real-Time PCR system (Thermo Fisher Scientific, Inc.) using the Takara SYBR ${ }^{\circledR}$ Premix Ex Taq ${ }^{\mathrm{TM}}$ kit to quantify the expression of target genes. Primers used in qPCR experiments are presented in Table I, with GAPDH serving as an internal reference. The thermal cycling conditions for qPCR were as follows: Holding stage conducted at $95^{\circ} \mathrm{C}$ for $30 \mathrm{sec}$; cycling stage conducted at $95^{\circ} \mathrm{C}$ for $5 \mathrm{sec}$ and $60^{\circ} \mathrm{C}$ for $34 \mathrm{sec}$ for 40 cycles; melt curve stage conducted at $95^{\circ} \mathrm{C}$ for $15 \mathrm{sec}, 60^{\circ} \mathrm{C}$ for $60 \mathrm{sec}, 95^{\circ} \mathrm{C}$ for $30 \mathrm{sec}$ and $60^{\circ} \mathrm{C}$ for $15 \mathrm{sec}$. Following normalization to the GAPDH gene, the expression of each target gene was calculated using the comparative cycle quantification $(\mathrm{Cq})$ method (24). In correlation analysis, the $\Delta \mathrm{Cq}$ values were calculated according to the following formula: $\Delta \mathrm{Cq}=\mathrm{Cq}$ (gene of interest)-Cq (GAPDH). For determination of the relative expression, the $2^{-\Delta \Delta \mathrm{Cq}}$ value was calculated according to the following formula: $\Delta \Delta \mathrm{Cq}=\Delta \mathrm{Cq}$ (control group) $-\Delta \mathrm{Cq}$ (experimental group).

Western blotting. A549 and HCT116 cells were treated with SAHA $(0.5 \mu \mathrm{M})$ or TSA $(100 \mathrm{nM})$ at $37^{\circ} \mathrm{C}$ for $24 \mathrm{~h}$. DMSO treatment was used as the control. A549 and HCT116 cells were washed three times with ice-cold PBS and subsequently lysed using western blotting lysis buffer, containing $50 \mathrm{mM}$ Tris- $\mathrm{HCl}$ (pH 7.6), $150 \mathrm{mM} \mathrm{NaCl}, 1 \mathrm{mM}$ EDTA, $1 \%$ NP-40, $0.5 \% \mathrm{Na}$-deoxycholate, $5 \mu \mathrm{g} / \mathrm{ml}$ aprotinin, $5 \mu \mathrm{g} / \mathrm{ml}$ leupeptin and $1 \mathrm{mM}$ phenylmethylsulphonyl fluoride. Cell lysates were cleared by centrifugation at $12,000 \mathrm{x} \mathrm{g}$ at $4^{\circ} \mathrm{C}$ for $30 \mathrm{~min}$ and denatured by boiling in Laemmli buffer. Bovine serum albumin was used as the standard for determining the protein concentration using a Bradford protein assay (Bio-Rad Laboratories, Inc., Hercules, CA, USA) Equal amounts of protein samples were loaded onto $8 \%$ gels and separated by SDS-PAGE, following which they were electrophoretically transferred onto polyvinylidene difluoride (PVDF) membranes. Following blocking with 5\% non-fat milk for $2 \mathrm{~h}$ at room temperature, the PVDF membranes were incubated with primary antibodies (ABCB1, ABCC1, ABCC2, ABCC4, ABCC5, ABCC6, ABCG2, $\alpha$-tubulin, HDAC1, HDAC2, HDAC3 and HDAC4 at a dilution of 1:1,000; $\mathrm{ABCC} 3, \mathrm{ABCC} 10, \mathrm{ABCC} 11$ and $\mathrm{ABCC} 12$ at a dilution of $1: 50)$ at $4^{\circ} \mathrm{C}$ overnight. Membranes were then incubated with 
Table I. Primers used in reverse transcription-quantitative polymerase chain reaction assay.

\begin{tabular}{lll} 
Gene & \multicolumn{1}{c}{ Forward primer $\left(5^{\prime}-3^{\prime}\right)$} & \multicolumn{1}{c}{ Reverse primer $\left(5^{\prime}-3^{\prime}\right)$} \\
\hline ABCB1 & TGCTCAGACAGGATGTGAGTTG & AATTACAGCAAGCCTGGAACC \\
ABCC 1 & GCCAAGAAGGAGGAGACC & AGGAAGATGCTGAGGAAGG \\
ABCC2 & TGGTGGCAACCTGAGCATAGG & ACTCGTTTTGATGGTCGTCTG \\
ABCC3 & CTTAAGACTTCCCCTCAACATGC & GGTCAAGTTCCTCTTGGCTC \\
ABCC4 & GGTTCCCCTTGGAATCATTT & AATCCTGGTGTGCATCAAACAG \\
ABCC5 & ACCCGTTGTTGCCATCTTAG & GCTTTGACCCAGGCATACAT \\
ABCC6 & GTGGTGTTTGCTGTCCACAC & ACGACACCAGGGTCAACTTC \\
ABCC10 & ATTGCCCATAGGCTCAACAC & AGCAGCCAGCACCTCTGTAT \\
ABCC11 & GGCTGAGCTACTGGTTGGAG & TGGTGAAAATCCCTGAGGAG \\
ABCC12 & GGTGTTCATGCTGGTGTTTGG & GCTCGTCCATATCCTTGGAA \\
ABCG2 & TATAGCTCAGATCATTGTCACAGTC & GTTGGTCGTCAGGAAGAAGAG \\
GAPDH & GCACCGTCAAGGCTGAGAAC & TGGTGAAGACGCCAGTGGA
\end{tabular}

ABC, ATP-binding cassette.

HRP-conjugated secondary antibodies (1:5,000 dilution) for $1.5 \mathrm{~h}$ at room temperature. Specific immune complexes were detected using Western Blotting Plus Chemiluminescence Reagent (Thermo Fisher Scientific, Inc.). Band intensity was quantified by densitometry analysis using Image-Pro Plus version 4.5 software (Media Cybernetics, Inc., Rockville, MD, USA).

Cell Counting Kit (CCK)-8 assay. Cell viability was measured using a CCK-8 assay. Briefly, A549 and HCT116 cells $\left(1 \times 10^{4} /\right.$ well $)$ were seeded into $96-$-well plates in medium supplemented with 10\% FBS (Gibco; Thermo Fisher Scientific, Inc.). The cells were treated with different concentrations of SAHA $(0,0.25,0.51,2$ and $4 \mu \mathrm{M})$ or TSA $(0,50,100,200$, 400 and $800 \mathrm{nM}$ ) at $37^{\circ} \mathrm{C}$ for $48 \mathrm{~h}$. Then, $10 \mu \mathrm{lCCK}-8$ solution (Dojindo Molecular Technologies, Inc., Kumamoto, Japan) was added to $100 \mu \mathrm{l}$ medium. The cells were incubated with CCK-8 in medium at $37^{\circ} \mathrm{C}$ for $2 \mathrm{~h}$, and the absorbance was then measured at $450 \mathrm{~nm}$ using a microplate reader (Bio-Rad Laboratories, Inc.).

To investigate the effect of SAHA and TSA on chemoresistance, HCT116 cells were treated with SAHA $(0.5 \mu \mathrm{M})$ or TSA $(100 \mathrm{nM})$ at $37^{\circ} \mathrm{C}$ for $24 \mathrm{~h}$, then the media were removed and the cells were treated with different concentrations of oxaliplatin $(0,5,10,20,40$ and $80 \mu \mathrm{g} / \mathrm{ml})$ or 5 -fluorouracil $(0,2.5,5,10,20$ and $40 \mu \mathrm{g} / \mathrm{ml})$ at $37^{\circ} \mathrm{C}$ for $48 \mathrm{~h}$. DMSO treatment was used as a control. The cells were diluted with $10 \mu \mathrm{l}$ CCK- 8 in medium at $37^{\circ} \mathrm{C}$ for $2 \mathrm{~h}$, and the absorbance was then measured at $450 \mathrm{~nm}$ using a microplate reader.

Statistical analysis. Data are presented as the mean \pm standard deviation of three independent experiments. One-way analysis of variance was used to assess the differences among multiple groups. Data were analyzed using two-tailed unpaired Student's t-tests for differences between any two groups. These analyses were performed using GraphPad Prism software, version 5.0 (GraphPad Software, Inc., La Jolla, CA, USA). P<0.05 was considered to indicate a statistically significant difference.

\section{Results}

HDAC inhibitors induce drug resistance in HCT116 cells. HDAC inhibitors are known to have a potent anticancer activity. The present study attempted to investigate the effect of two HDAC inhibitors, SAHA and TSA, on the expression of $\mathrm{ABC}$ transporters and drug resistance in cancer cells. The concentration of SAHA and TSA used to treat cancer cells should have no significant effect on cell survival. In order to determine the appropriate concentration, SAHA and TSA at concentrations of $0-4 \mu \mathrm{M}$ and $0-800 \mathrm{nM}$, respectively, were used in the cell viability assay, which are below their $\mathrm{IC}_{50}$ values. HCT116 and A549 cells were treated with different concentrations of SAHA or TSA for $48 \mathrm{~h}$, and the cell viability was assessed using a CCK-8 assay. As shown in Fig. 1A, slight inhibition was observed for concentrations $>0.5 \mu \mathrm{M}$ for SAHA and $>100 \mathrm{nM}$ for TSA. Therefore, $0.5 \mu \mathrm{M}$ SAHA and $100 \mathrm{nM}$ TSA were selected for use in subsequent experiments.

Next, the study investigated whether HDAC inhibitors affect drug resistance by performing a CCK-8 assay. HCT116 cells were pretreated with $0.5 \mu \mathrm{M}$ SAHA or $100 \mathrm{nM}$ TSA for $24 \mathrm{~h}$, following which cells were exposed to various concentrations of oxaliplatin or 5-fluorouracil for $48 \mathrm{~h}$, and cell viability was assessed using a CCK- 8 assay. The results revealed that, compared with the DMSO-treated group, SAHA and TSA pretreatment significantly decreased the sensitivity of HCT116 cells to anticancer drugs (Fig. 1B).

Effect of HDAC inhibitors on $A B C B 1, A B C C 5, A B C C 6$, $A B C C 10, A B C C 11$ and $A B C C 12$ expression. ABC transporter-induced drug efflux is closely associated with acquisition of chemo-resistance in cancer cells (3). To investigate the effect of HDAC inhibitors on the expression of $\mathrm{ABC}$ transporters, HCT116 and A549 cells were treated with $0.5 \mu \mathrm{M}$ SAHA and $100 \mathrm{nM}$ TSA for $24 \mathrm{~h}$. The mRNA and protein expression levels of various ABC transporters were detected using RT-qPCR and western blotting, respectively. The RT-qPCR results indicated that SAHA markedly upregulated 

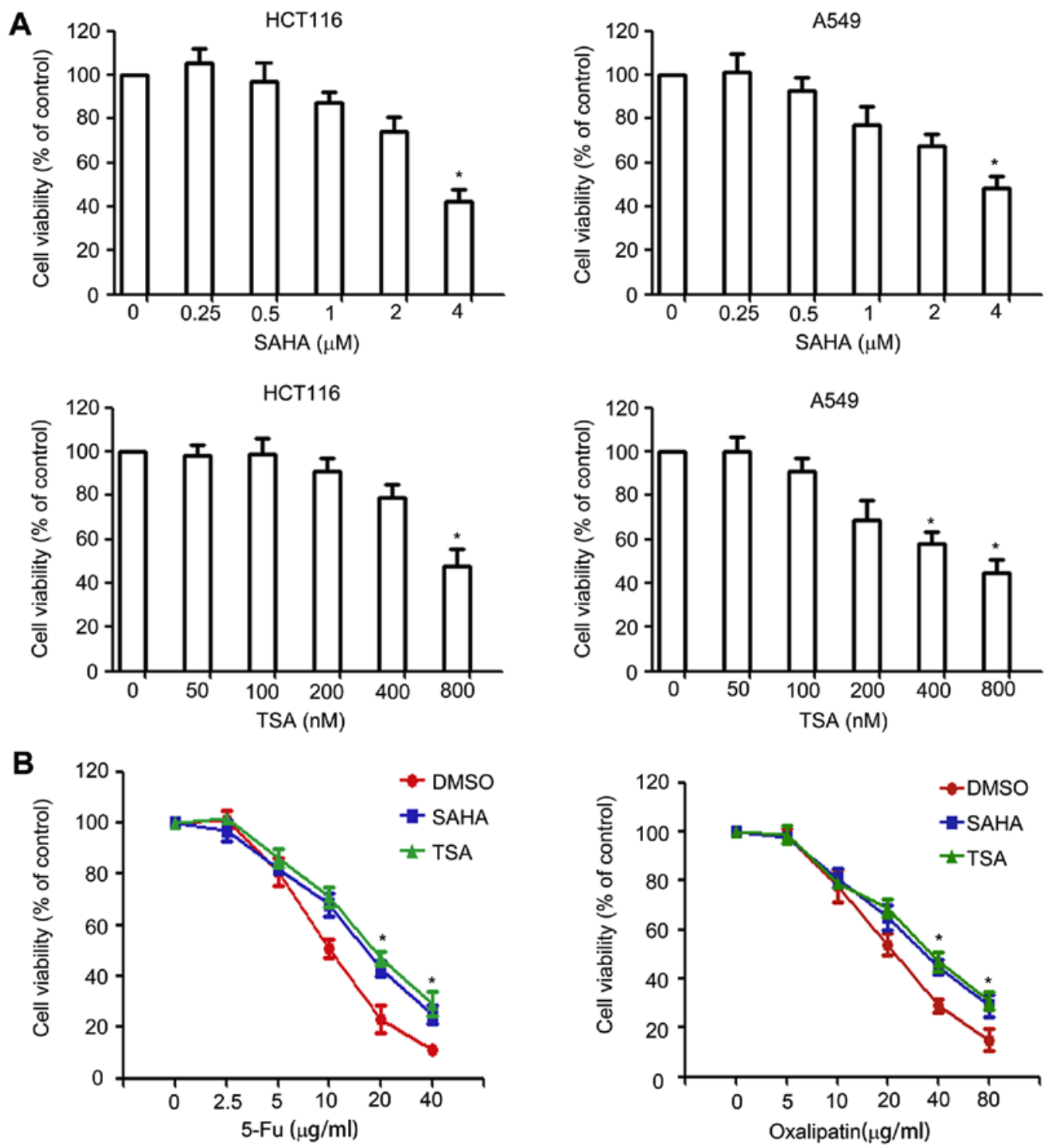

Figure 1. Histone deacetylase inhibitors induce drug resistance in HCT116 cells. (A) A549 and HCT116 cells were treated with various concentrations of SAHA or TSA for $48 \mathrm{~h}$, and cell viability was detected using a CCK-8 assay. (B) HCT116 cells were pretreated with SAHA (0.5 $\mu$ M) or TSA (100 nM) for $24 \mathrm{~h}$. Subsequently, the media were removed and the cells were treated with various concentrations of 5-Fu or oxaliplatin for $48 \mathrm{~h}$. Cell viability was assessed using a CCK-8 assay. " $\mathrm{P}<0.05$ vs. corresponding control group. ABC, ATP-binding cassette; SAHA, suberoylanilide hydroxamic acid; TSA, trichostatin A; CCK-8, Cell Counting Kit-8; 5-Fu, 5-fluorouracil.

mRNA expression of ABCB1, ABCC5, ABCC10, $\mathrm{ABCC} 11$ and $\mathrm{ABCC} 12$ in $\mathrm{A} 549$ cells and $\mathrm{ABCB} 1, \mathrm{ABCC} 10$ and ABCC12 in HCT116 cells. TSA markedly upregulated mRNA levels of ABCB1, ABCC5, ABCC6, ABCC10, ABCC11 and ABCC12 in A549 cells and HCT116 cells (Fig. 2A). Similarly, the results of western blotting revealed that SAHA and TSA upregulated $\mathrm{ABCB} 1, \mathrm{ABCC} 10, \mathrm{ABCC} 11$ and $\mathrm{ABCC} 12$ protein expression. However, SAHA and TSA had no clear effect on the expression levels of ABCC5 and ABCC6 (Fig. 2B).

Effect of HDAC inhibitors on ABCC1, ABCC2, ABCC3, $A B C C 4$ and $A B C G 2$ expression. Treatment with HDAC inhibitors affected the expression of $\mathrm{ABCC} 1, \mathrm{ABCC} 2, \mathrm{ABCC} 2$ and $\mathrm{ABCC} 4$ differently, as compared with $\mathrm{ABCB} 1, \mathrm{ABCC} 5, \mathrm{ABCC} 6$, $\mathrm{ABCC} 10, \mathrm{ABCC} 11$ and $\mathrm{ABCC} 12$. It was observed that SAHA and TSA treatment downregulated the expression levels of ABCC1, ABCC3 and ABCC4 mRNA (Fig. 3A). Similarly, the western blotting results indicated that the $\mathrm{ABCC} 1$ and $\mathrm{ABCC} 3$ protein expression levels were also decreased (Fig. 3B). Notably, while SAHA and TSA decreased ABCC 2 mRNA expression, its protein expression in A549 and HCT116 cells was found to be increased (Fig. 3A and B). SAHA and TSA exerted no obvious effects on the protein expression levels of ABCC4 (Fig. 3B).

The effect of HDAC inhibitors on ABCG2 expression was further investigated in A549 and HCT116 cells. As shown in Fig. 3A, SAHA and TSA downregulated ABCG2 mRNA expression in A549 cells, while no marked effect was observed in HCT116 cells. Furthermore, western blotting revealed that SAHA and TSA had no evident effect on the expression of ABCG2 protein in A549 and HCT116 cells (Fig. 3B).

Effect of HDAC inhibitors on the expression of HDAC1, HDAC2, HDAC3 and HDAC4. Since HDACs serve an important role in the regulation of gene transcription, the effect of HDAC 
A
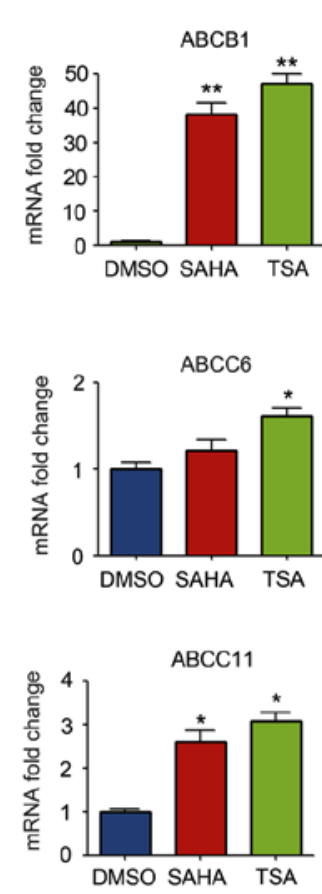

B

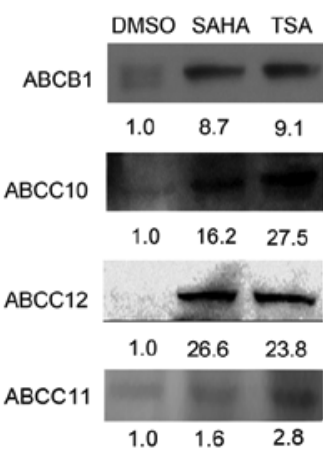

ABCC5

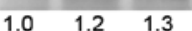

ascos

$\begin{array}{lll}1.0 & 1.0 & 0.9\end{array}$

$\alpha$-Tubulin
A549
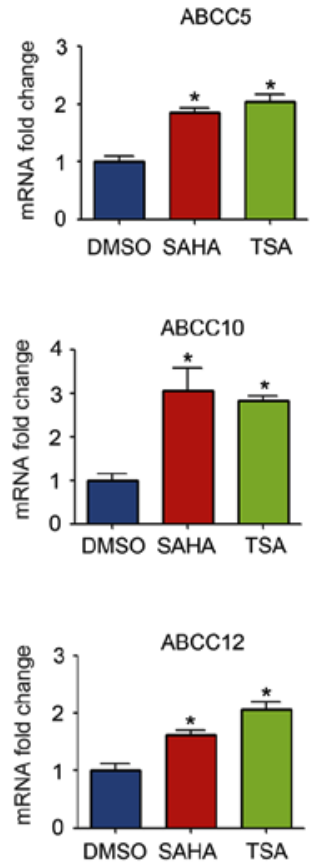

HCT116
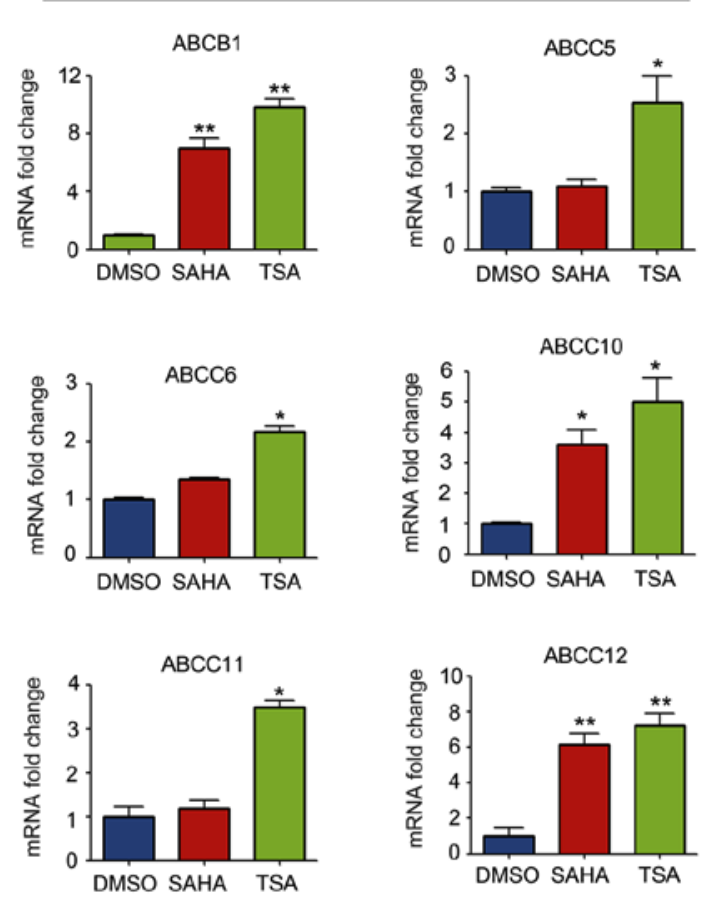

DMSO SAHA TSA
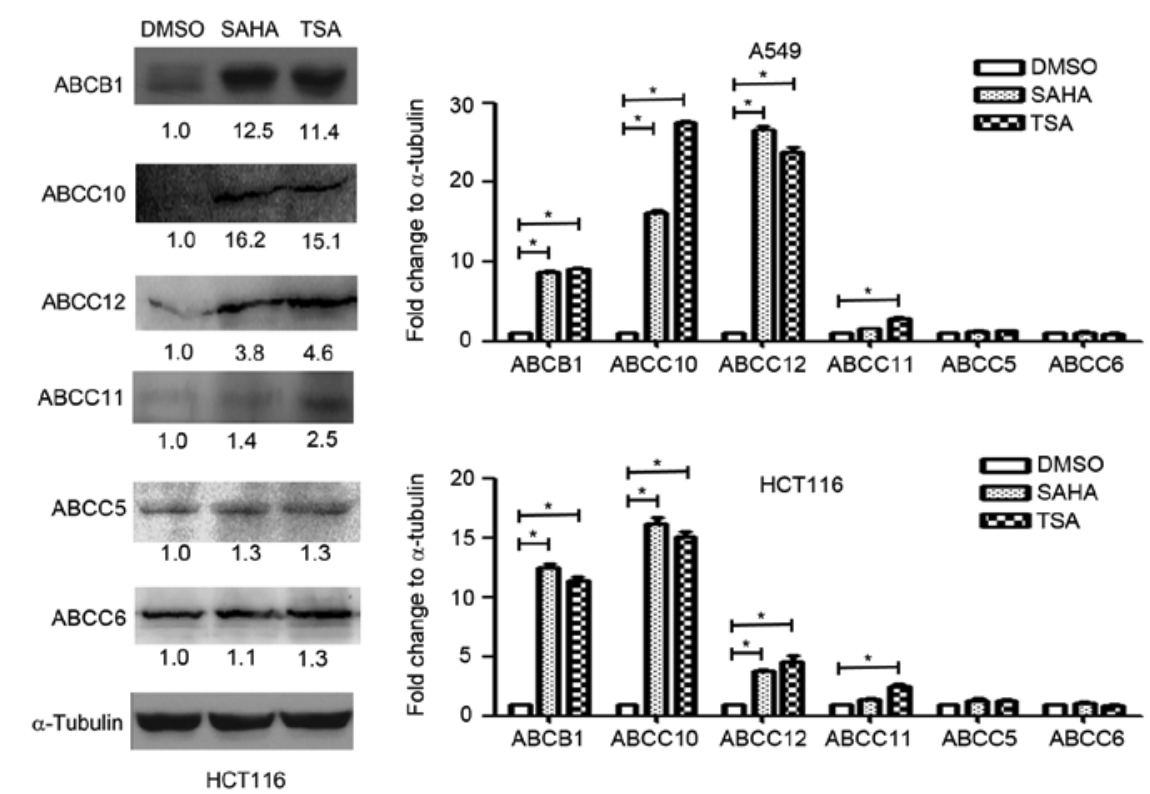

Figure 2. Histone deacetylase inhibitors increased the ABCB1, ABCC5, ABCC6, ABCC10, ABCC11 and ABCC12 expression levels. A549 and HCT116 cells were treated with DMSO, SAHA $(0.5 \mu \mathrm{M})$ or TSA $(100 \mathrm{nM})$ for $24 \mathrm{~h}$. (A) mRNA expression levels of ABCB1, ABCC5, ABCC6, ABCC10, ABCC11 and $\mathrm{ABCC} 12$ were measured using reverse transcription-quantitative polymerase chain reaction. (B) Protein expression levels of $\mathrm{ABCB} 1, \mathrm{ABCC} 5, \mathrm{ABCC} 6$, $\mathrm{ABCC} 10, \mathrm{ABCC} 11$ and $\mathrm{ABCC} 12$ were detected using western blotting. ${ }^{*} \mathrm{P}<0.05$ and ${ }^{* *} \mathrm{P}<0.01$, vs. DMSO group. ABC, ATP-binding cassette; SAHA, suberoylanilide hydroxamic acid; TSA, trichostatin A.

inhibitors on HDAC1, HDAC2, HDAC3 and HDAC4 expression was measured in A549 and HCT116 cells. HDAC1-4 exert their function depending on the protein; therefore, protein expression was detected by western blotting. As shown in Fig. 4, SAHA and TSA decreased the protein expression levels of HDAC 3 and HDAC4, compared with the DMSO-treated group. By contrast, SAHA and TSA had no marked effects on HDAC1 and HDAC2 expression levels in A549 and HCT116 cells (Fig. 4).

\section{Discussion}

Chemotherapy is a common strategy used for the treatment of malignant tumors. However, the efficacy of chemotherapy is limited by the development of an MDR phenotype due to overexpression of $\mathrm{ABC}$ transporters in cancer cells. Chemoresistance-associated $\mathrm{ABC}$ transporters mainly include ABCB1, ABCC1-6, ABCC10-12 and ABCG2 (3). 
A
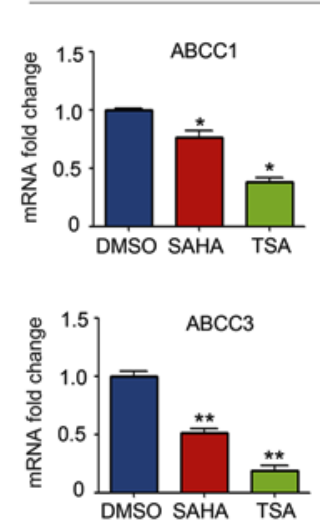

ABCG2

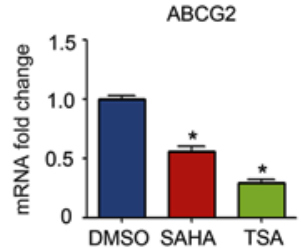

B

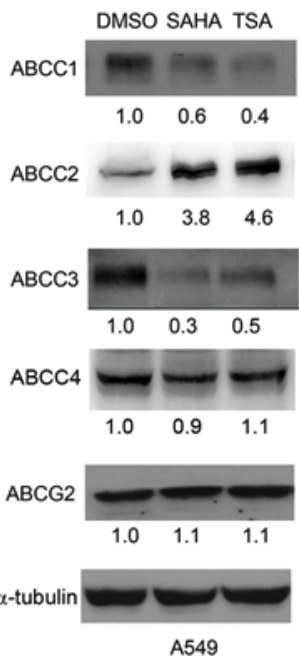

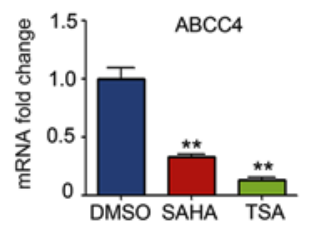

A549
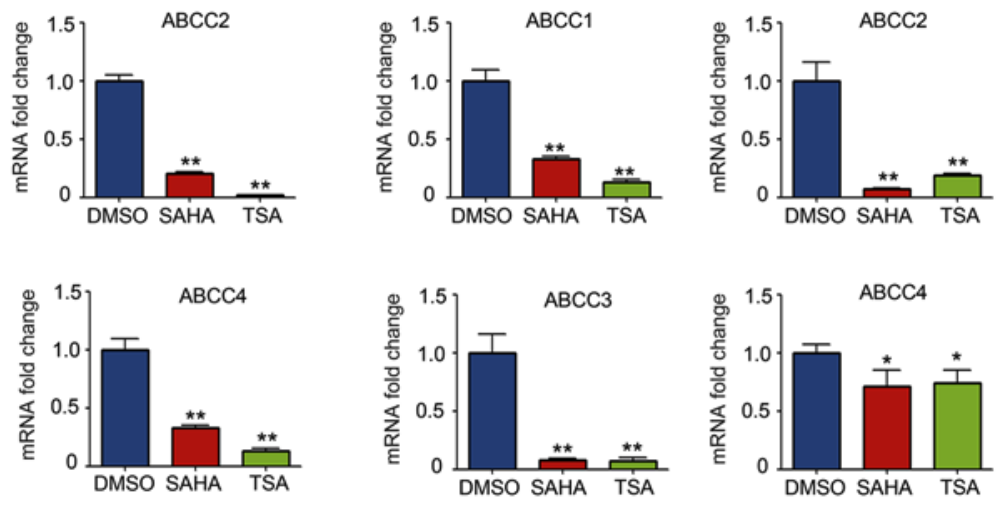

ABCG2

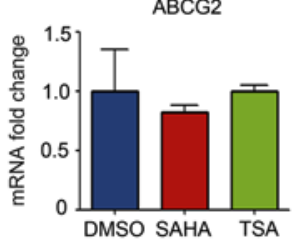

HCT116
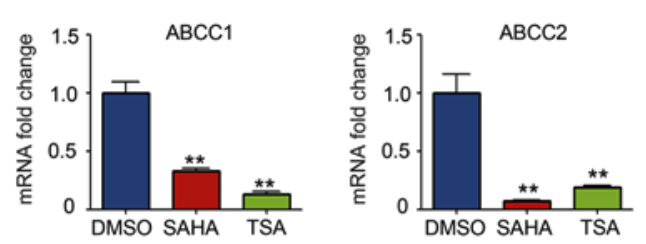$$
\text { 西 }
$$

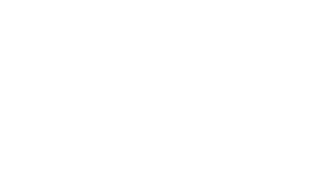

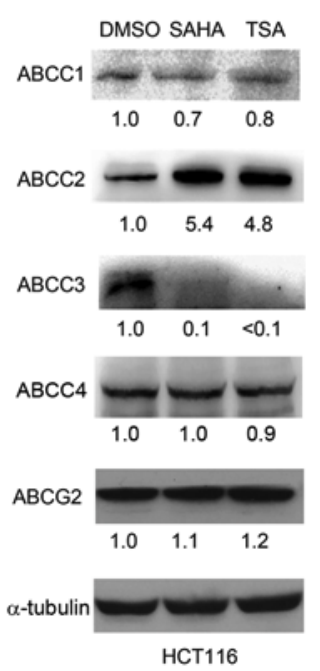
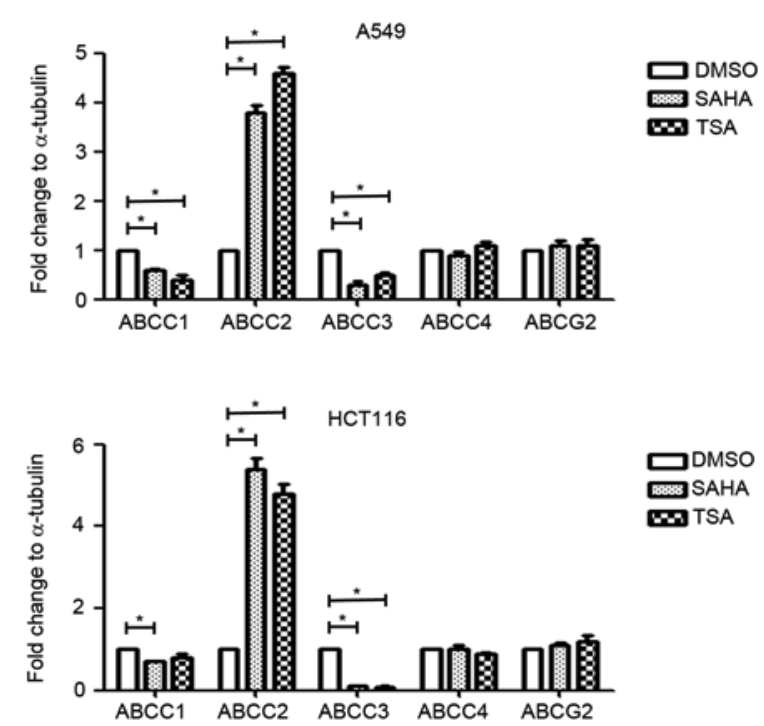

Figure 3. Effect of histone deacetylase inhibitors on ABCC1, ABCC2, ABCC3, ABCC4 and ABCG2 expression levels. A549 and HCT116 cells were treated with DMSO, SAHA $(0.5 \mu \mathrm{M})$ or TSA $(100 \mathrm{nM})$ for $24 \mathrm{~h}$. (A) mRNA and (B) protein expression levels of ABCC1, ABCC2, ABCC3, ABCC4 and ABCG2 were measured using reverse transcription-quantitative polymerase chain reaction and western blotting, respectively. ${ }^{*} \mathrm{P}<0.05$ and ${ }^{* *} \mathrm{P}<0.01$, vs. DMSO group. ABC, ATP-binding cassette; SAHA, suberoylanilide hydroxamic acid; TSA, trichostatin A.

These transporters function as efflux pumps that energetically translocate chemotherapeutic drugs from inside to outside of cancer cells, reducing the intracellular drug concentration and resulting in an MDR phenotype (3). In order to overcome the MDR phenotype induced by HDAC inhibitors, it is crucial to understand the effect of HDAC inhibitors on the expression of chemoresistance-associated ABC transporters. In the present study, it was demonstrated that treatment with HDAC inhibitors increased the expression levels of ABCB1, ABCC2, ABCC5, ABCC6, ABCC10, ABCC11 and ABCC12 in cancer cells, whereas it decreased the expression levels of $\mathrm{ABCC} 1, \mathrm{ABCC} 3$ and $\mathrm{ABCC} 4$. To the best of our knowledge, this is the first systematic investigation on the effect of HDAC inhibitors on the expression of chemoresistance-associated ABC transporters.
ABCB1, the most well-known ABC transporter, extrudes natural toxins, anticancer drugs and drug metabolites across the plasma membrane, conferring an MDR phenotype in various cancer cells $(25,26)$. A number of studies have reported that HDAC inhibitors may promote ABCB1 expression in several types of cancer cells $(27,28)$. For instance, the HDAC inhibitors SAHA, TSA and phenylbutyrate were reported to increase ABCB1 expression in acute myeloid leukemia (29). Our previous study revealed that SAHA, TSA and sodium butyrate induced ABCB1 expression by transcriptional activation of STAT3, and stabilized ABCB1 mRNA in lung and colorectal cancer $(22,23)$. Consistently, the present study also demonstrated that SAHA and TSA significantly increase ABCB1 mRNA and protein expression 

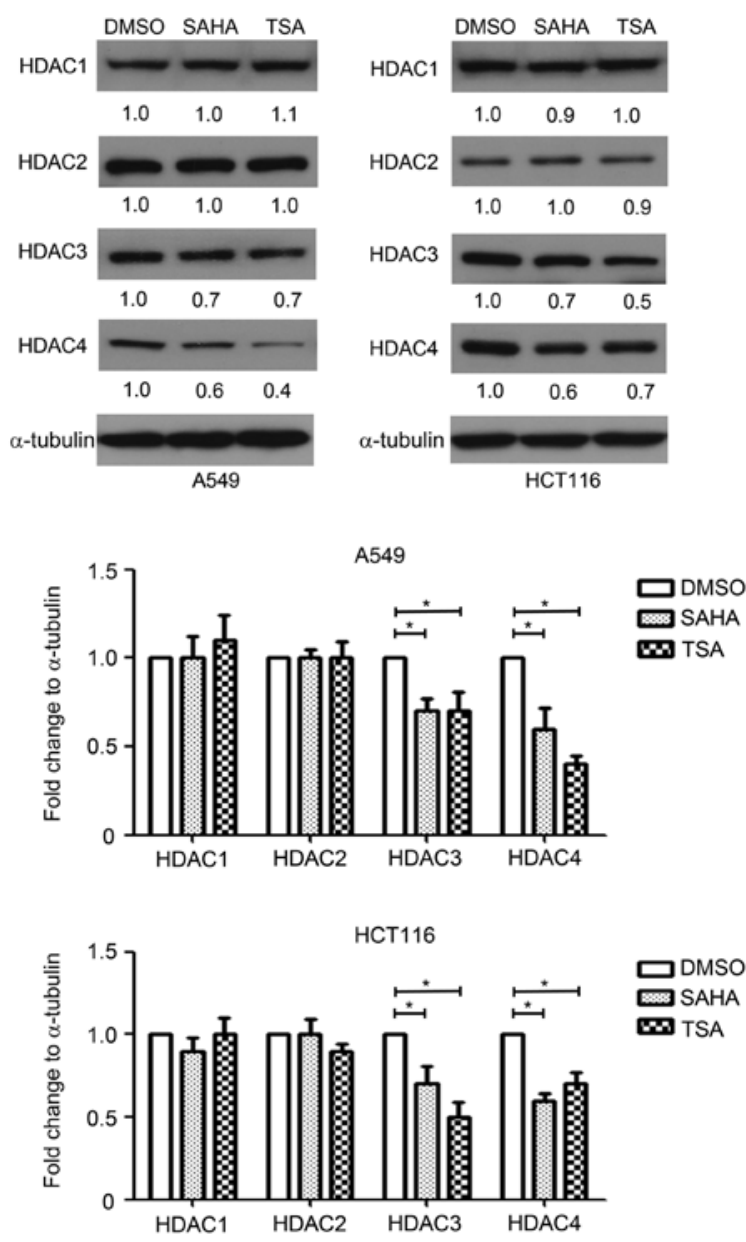

Figure 4. Effect of HDAC inhibitors on the HDAC1, HDAC2, HDAC3 and HDAC4 expression levels. A549 and HCT116 cells were treated with DMSO, SAHA $(0.5 \mu \mathrm{M})$ or TSA $(100 \mathrm{nM})$ for $24 \mathrm{~h}$, following which HDAC1, HDAC2, HDAC3 and HDAC4 expression was detected using western blotting. ${ }^{*} \mathrm{P}<0.05$ vs. DMSO group. HDAC, histone deacetylase; SAHA, suberoylanilide hydroxamic acid; TSA, trichostatin A.

in A549 and HCT116 cells. Recent research has suggested that continuous stimulation with the HDAC inhibitor FK228 may active $\mathrm{ABCG} 2$, another $\mathrm{ABC}$ transporter, at the mRNA and protein levels in renal and colon cancer cells to give an MDR phenotype (30). Furthermore, SAHA, TSA and phenylbutyrate have been reported to increase ABCG2 expression in acute myeloid leukemia $(19,29)$. However, in the present study it was observed that SAHA decreased ABCG2 mRNA expression in A549 cells, while neither SAHA nor TSA had any evident influence on ABCG2 protein expression in A549 and HCT116 cells.

ABCC members have been demonstrated to confer chemoresistance in cancer cells by translocating a variety of structurally diverse glutathione conjugates or therapeutic drugs (4). A previous study suggested that SAHA, TSA and valproate were able to increase $\mathrm{ABCC} 11$ expression by promoting its transcription in acute myeloid leukemia (29). In the present study, the data revealed that SAHA and TSA increased ABCC5, ABCC6, ABCC10, ABCC11 and ABCC12 mRNA expression levels, as well as ABCC10, $\mathrm{ABCC} 12$ protein expression levels. By contrast, SAHA and TSA significantly decreased $\mathrm{ABCC} 1, \mathrm{ABCC} 3$ and $\mathrm{ABCC} 4$ mRNA expression levels, as well as $\mathrm{ABCC} 1$ and $\mathrm{ABCC} 3$ protein levels. A recent study reported that the HDAC inhibitors SAHA and belinostat downregulated $\mathrm{ABCC} 1$ and upregulated $\mathrm{ABCB} 1$ expression in T-cell lymphoma and T-cell prolymphocytic leukemia (31).

Of the nine $\mathrm{ABCC}$ proteins investigated in the present study, ABCC4, ABCC5, ABCC11 and ABCC12 have a typical ABC structure, with four domains (MSD1, MSD2, NBD1 and NBD2). The other ABCCs, namely ABCC1, ABCC2, ABCC3, ABCC6 and ABCC10 have an additional fifth domain, MSD0 (4). It can thus be speculated that HDAC inhibitors may induce different effects on ABCC expression in cancer cells due to their different structures. Notably, the current study results revealed that SAHA and TSA significantly decreased ABCC2 mRNA expression, but evidently increased its protein expression in A549 and HCT116 cells. Post-transcriptional regulation, such as mRNA stability alternations and protein stability modifications, serves an important role in protein expression $(20,32,33)$. Therefore, HDAC inhibitors may influence the post-transcriptional regulation processes that regulate ABCC 2 mRNA and protein expression.

According to the findings of the current study, it can be speculated that the underlying mechanisms responsible for the differential effects of HDAC inhibitors on ABC transporters may be associated with differences in site-specific acetylation/methylation of histones. For instance, acetylation and mono-methylation of histone 3 at Lys-9 activates gene transcription, whereas di- and tri-methylation of the same residue results in gene suppression (34-36). Several HDAC family members are aberrantly expressed in various cancer types and may have potential as target molecules for anticancer treatments (37). Valdez et al (31) also reported that the HDAC inhibitors SAHA, TSA and phenylbutyrate downregulated the expression levels of HDAC3, HDAC4 and HDAC6 in T-cell lymphoma and T-cell prolymphocytic leukemia. In the present study, it was observed that SAHA and TSA treatment decreased HDAC3 and HDAC4 expression levels, but had no significant effect on HDAC1 or HDAC2 expression. Furthermore, an earlier study indicated that the HDAC3 and HDAC4 complex stimulated the transcriptional activity of mineralocorticoid receptor (MR), and HDAC4 served an important role as a scaffold between MR and HDAC3 (38). Acetylation occurs, in part, due to decreased HDAC3 and HDAC4 protein expression, while methylation is most likely to occur due to the functional interaction between histone methyltransferases and deacetylases (39). Nevertheless, it remains unclear which histone modifications contribute to the differential effects of HDAC inhibitors on the expression of ABC transporters.

$\mathrm{ABC}$ transporters have different substrates; for instance, $\mathrm{ABCC} 1$ is known to pump GSH-conjugated DNA alkylators out of cells $(4,40)$, while decreased ABCC1 activity may cause cellular accumulation of DNA alkylating agents and thus enhanced cytotoxicity. Cancer cells with high levels of ABCC1 are more resistant to DNA alkylating agents, such as busulfan and chlorambucil. It has been reported that busulfan exerts synergistic cytotoxicity when used in combination with HDAC inhibitors $(41,42)$. However, the present study demonstrated that SAHA and TSA increased ABCB1 expression, which is known to pump its substrates, such as doxorubicin, vincristine and prednisone, out of cells $(25,30)$. The increased 
ABCB1 expression induced by HDAC inhibitors may lead to reduced anticancer activity of ABCB1 substrates. Therefore, the results of the present study indicate that it is important to select appropriate drugs in combination with HDAC inhibitors.

In conclusion, the present study demonstrated that HDAC inhibitors have different effects on the expression of $\mathrm{ABC}$ transporters in A549 and HCT116 cells. SAHA and TSA increased the expression levels of ABCB1, ABCC2, ABCC5, $\mathrm{ABCC} 6, \mathrm{ABCC} 10, \mathrm{ABCC} 11$ and $\mathrm{ABCC} 12$, whereas they downregulated $\mathrm{ABCC} 1, \mathrm{ABCC} 3$ and $\mathrm{ABCC} 4$. Furthermore, SAHA and TSA induced drug resistance in HCT116 cells, and decreased HDAC3 and HCAC4 expression levels. In future studies, drug resistance mediated by SAHA and TSA in A549 cells will be investigated. ABC transporters have different substrates (14). Differential effects of HDAC inhibitors on the expression of drug transporters support the necessity for caution in combining these drugs with other chemotherapeutic agents. The present study seems timely in light of an ongoing clinical trial (no. NCT01280526) testing the value of combining romidepsin, a HDAC inhibitor, with cyclophosphamide, doxorubicin, vincristine and prednisone (31). Particularly, efflux of doxorubicin, vincristine and prednisone may increase with HDAC inhibitor-mediated upregulation of ABCB1 (43). The present study highlighted the importance of understanding the mechanism of drug combination to achieve more efficient cytotoxicity to cancer cells. However, the molecular mechanisms of the different effects of $\mathrm{ABC}$ transporter expression induced by HDAC inhibitors remain unclear. It is necessary to investigate the underlying mechanisms in future studies.

\section{Acknowledgements}

Not applicable.

\section{Funding}

The present study was funded by the National Natural Science Foundation of China (grant no. 81502599), the Natural Science Foundation of Anhui Province (grant no. 1608085QH217), the Natural Science Foundation of Shandong Province (grant no. ZR2013HM43), the China Postdoctoral Science Foundation funded project (grant no. 2016M592040) and the Anhui Province Postdoctoral Science Foundation funded project (grant no. 2016B142).

\section{Availability of data and materials}

The datasets used and/or analyzed during the present study are available from the corresponding author on reasonable request.

\section{Authors' contributions}

HW contributed to the study design, data acquisition and analysis, and drafted the manuscript. BJW participated in the study design, data acquisition and revision of the manuscript. $\mathrm{CHC}, \mathrm{YZ}, \mathrm{BS}$ and RJ assisted in the performance of the statistical analysis. All authors have read and approved the final manuscript.

\section{Ethics approval and consent to participate}

Not applicable.

\section{Patient consent for publication}

Not applicable.

\section{Competing interests}

The authors declare that they have no competing interests.

\section{References}

1. Mohammad IS, He W and Yin L: Understanding of human ATP binding cassette superfamily and novel multidrug resistance modulators to overcome MDR. Biomed Pharmacother 100: 335-348, 2018.

2. Wang L, Tan RZ, Zhang ZX, Yin R, Zhang YL, Cui WJ and He T: Association between Twist and multidrug resistance gene-associated proteins in Taxol ${ }^{\circledR}$-resistant MCF-7 cells and a 293 cell model of Twist overexpression. Oncol Lett 15: 1058-1066, 2018.

3. Fukuda Y and Schuetz JD: ABC transporters and their role in nucleoside and nucleotide drug resistance. Biochem Pharmacol 83: 1073-1083, 2012.

4. Chen ZS and Tiwari AK: Multidrug resistance proteins (MRPs/ABCCs) in cancer chemotherapy and genetic diseases. FEBS J 278: 3226-3245, 2011.

5. Liu YR, Liang L, Zhao JM, Zhang Y, Zhang M, Zhong WL, Zhang Q, Wei JJ, Li M, Yuan J, et al: Twist1 confers multidrug resistance in colon cancer through upregulation of ATP-binding cassette transporters. Oncotarget 8: 52901-52912, 2017.

6. Norouzi-Barough L, Sarookhani M, Salehi R, Sharifi M and Moghbelinejad S: CRISPR/Cas9, a new approach to successful knockdown of ABCB1/P-glycoprotein and reversal of chemosensitivity in human epithelial ovarian cancer cell line. Iran J Basic Med Sci 21: 181-187, 2018.

7. Ceballos MP, Rigalli JP, Cere LI, Semeniuk M, Catania VA and Ruiz ML: ABC transporters: Regulation and association with multidrug resistance in hepatocellular carcinoma and colorectal carcinoma. Curr Med Chem, 2018.

8. Maia RC, Vasconcelos FC, Souza PS and Rumjanek VM: Towards comprehension of the ABCB1/P-glycoprotein role in chronic myeloid leukemia. Molecules 23, 2018.

9. Assaraf YG: Molecular basis of antifolate resistance. Cancer Metastasis Rev 26: 153-181, 2007.

10. Borst P, Evers R, Kool M and Wijnholds J: A family of drug transporters: The multidrug resistance-associated proteins. J Natl Cancer Inst 92: 1295-1302, 2000.

11. Dean M, Hamon Y and Chimini G: The human ATP-binding cassette (ABC) transporter superfamily. J Lipid Res 42: 1007-1017, 2001.

12. Wang J, Yunyun Z, Wang L, Chen X and Zhu Z: ABCG2 confers promotion in gastric cancer through modulating downstream CRKL in vitro combining with biostatistics mining. Oncotarget 8: 5256-5267, 2017.

13. Kaehler M, Ruemenapp J, Gonnermann D, Nagel I, Bruhn O, Haenisch S, Ammerpohl O, Wesch D, Cascorbi I and Bruckmueller H: MicroRNA-212/ABCG2-axis contributes to development of imatinib-resistance in leukemic cells. Oncotarget 8: 92018-92031, 2017.

14. Gottesman MM, Fojo T and Bates SE: Multidrug resistance in cancer: Role of ATP-dependent transporters. Nat Rev Cancer 2: 48-58, 2002.

15. Ling V: Multidrug resistance: Molecular mechanisms and clinical relevance. Cancer Chemother Pharmacol (40 Suppl): S3-S8, 1997.

16. Xu W, Liu H, Liu ZG, Wang HS, Zhang F, Wang H, Zhang J, Chen JJ, Huang HJ, Tan Y, et al: Histone deacetylase inhibitors upregulate Snail via Smad2/3 phosphorylation and stabilization of Snail to promote metastasis of hepatoma cells. Cancer Lett 420: 1-13, 2018.

17. Jiang GM, Wang HS, Zhang F, Zhang KS, Liu ZC, Fang R, Wang H, Cai SH and Du J: Histone deacetylase inhibitor induction of epithelial-mesenchymal transitions via up-regulation of Snail facilitates cancer progression. Biochim Biophys Acta 1833: 663-671, 2013. 
18. Botrugno OA, Santoro F and Minucci S: Histone deacetylase inhibitors as a new weapon in the arsenal of differentiation therapies of cancer. Cancer Lett 280: 134-144, 2009.

19. Ni X, Li L and Pan G: HDAC inhibitor-induced drug resistance involving ATP-binding cassette transporters (Review). Oncol Lett 9: 515-521, 2015.

20. Di Costanzo A, Del Gaudio N, Conte L, Dell'Aversana C, Vermeulen M, de Thé H, Migliaccio A, Nebbioso A and Altucci L: The HDAC inhibitor SAHA regulates CBX2 stability via a SUMO-triggered ubiquitin-mediated pathway in leukemia. Oncogene 37: 2559-2572, 2018.

21. Stimson L and La Thangue NB: Biomarkers for predicting clinical responses to HDAC inhibitors. Cancer Lett 280: 177-183, 2009.

22. Wang H, Huang C, Zhao L, Zhang H, Yang JM, Luo P, Zhan BX, Pan Q, Li J and Wang BL: Histone deacetylase inhibitors regulate P-gp expression in colorectal cancer via transcriptional activation and mRNA stabilization. Oncotarget 7: 49848-49858, 2016.

23. Zhao L, Bin S, He HL, Yang JM, Pu YC, Gao CH, Wang H and Wang BL: Sodium butyrate increases P-gp expression in lung cancer by upregulation of STAT3 and mRNA stabilization of ABCB1. Anticancer Drugs 29: 227-233, 2018.

24. Livak KJ and Schmittgen TD: Analysis of relative gene expression data using real-time quantitative PCR and the 2(-Delta Delta C(T)) method. Methods 25: 402-408, 2001

25. Sharom FJ: The P-glycoprotein multidrug transporter. Essays Biochem 50: 161-178, 2011.

26. Han Z and Shi L: Long non-coding RNA LUCAT1 modulates methotrexate resistance in osteosarcoma via miR-200c/ABCB1 axis. Biochem Biophys Res Commun 495: 947-953, 2018.

27. Robey RW, Zhan Z, Piekarz RL, Kayastha GL, Fojo T and Bates SE: Increased MDR1 expression in normal and malignant peripheral blood mononuclear cells obtained from patients receiving depsipeptide (FR901228, FK228, NSC630176). Clin Cancer Res 12: 1547-1555, 2006.

28. Tabe Y, Konopleva M, Contractor R, Munsell M, Schober WD, Jin L, Tsutsumi-Ishii Y, Nagaoka I, Igari J and Andreeff M: Up-regulation of MDR1 and induction of doxorubicin resistance by histone deacetylase inhibitor depsipeptide (FK228) and ATRA in acute promyelocytic leukemia cells. Blood 107: 1546-1554, 2006.

29. Hauswald S, Duque-Afonso J, Wagner MM, Schertl FM, Lübbert M, Peschel C, Keller U and Licht T: Histone deacetylase inhibitors induce a very broad, pleiotropic anticancer drug resistance phenotype in acute myeloid leukemia cells by modulation of multiple $\mathrm{ABC}$ transporter genes. Clin Cancer Res 15: 3705-3715, 2009

30. To KK, Polgar O, Huff LM, Morisaki K and Bates SE: Histone modifications at the ABCG2 promoter following treatment with histone deacetylase inhibitor mirror those in multidrug-resistant cells. Mol Cancer Res 6: 151-164, 2008.
31. Valdez BC, Li Y, Murray D, Brammer JE, Liu Y, Hosing C, Nieto Y, Champlin RE and Andersson BS: Differential effects of histone deacetylase inhibitors on cellular drug transporters and their implications for using epigenetic modifiers in combination chemotherapy. Oncotarget 7: 63829-63838, 2016.

32. Ménez C, Mselli-Lakhal L, Foucaud-Vignault M, Balaguer P, Alvinerie $\mathrm{M}$ and Lespine A: Ivermectin induces P-glycoprotein expression and function through mRNA stabilization in murine hepatocyte cell line. Biochem Pharmacol 83: 269-278, 2012.

33. Kim SH, Kang HJ, Na H and Lee MO: Trichostatin A enhances acetylation as well as protein stability of ERalpha through induction of p300 protein. Breast Cancer Res 12: R22, 2010.

34. Barski A, Cuddapah S, Cui K, Roh TY, Schones DE, Wang Z, Wei G, Chepelev I and Zhao K: High-resolution profiling of histone methylations in the human genome. Cell 129: 823-837, 2007.

35. Koch CM, Andrews RM, Flicek P, Dillon SC, Karaöz U, Clelland GK, Wilcox S, Beare DM, Fowler JC, Couttet P, et al: The landscape of histone modifications across $1 \%$ of the human genome in five human cell lines. Genome Res 17: 691-707, 2007.

36. Rosenfeld JA, Wang Z, Schones DE, Zhao K, DeSalle R and Zhang MQ: Determination of enriched histone modifications in non-genic portions of the human genome. BMC Genomics 10: $143,2009$.

37. Witt O, Deubzer HE, Milde T and Oehme I: HDAC family: What are the cancer relevant targets? Cancer Lett 277: 8-21, 2009.

38. Lee HA, Song MJ, Seok YM, Kang SH, Kim SY and Kim I: Histone deacetylase 3 and 4 complex stimulates the transcriptional activity of the mineralocorticoid receptor. PLoS One 10: e0136801, 2015.

39. Vaute O, Nicolas E, Vandel L and Trouche D: Functional and physical interaction between the histone methyl transferase Suv39H1 and histone deacetylases. Nucleic Acids Res 30: 475-481, 2002.

40. Johnson ZL and Chen J: ATP binding enables substrate release from multidrug resistance protein 1. Cell 172: 81-89.e10, 2018.

41. Valdez BC, Nieto Y, Murray D, Li Y, Wang G, Champlin RE and Andersson BS: Epigenetic modifiers enhance the synergistic cytotoxicity of combined nucleoside analog-DNA alkylating agents in lymphoma cell lines. Exp Hematol 40: 800-810, 2012.

42. Nieto Y, Valdez BC, Thall PF, Ahmed S, Jones RB, Hosing C, Popat U, Shpall EJ, Qazilbash M, Gulbis A, et al: Vorinostat combined with high-dose gemcitabine, busulfan, and melphalan with autologous stem cell transplantation in patients with refractory lymphomas. Biol Blood Marrow Transplant 21: 1914-1920, 2015.

43. Köck K, Grube M, Jedlitschky G, Oevermann L, Siegmund W, Ritter CA and Kroemer HK: Expression of adenosine triphosphate-binding cassette (ABC) drug transporters in peripheral blood cells: Relevance for physiology and pharmacotherapy. Clin Pharmacokinet 46: 449-470, 2007. 